\title{
So Many Options, Where Do We Start? An Overview of the Care Transitions Literature
}

\author{
Devan Kansagara, MD, MCR ${ }^{1,2,3 *}$, Joseph C Chiovaro, MD ${ }^{1,2}$, David Kagen, MD,2, Stephen Jencks, MD, MPH ${ }^{4}, \mathrm{Kerry} \mathrm{Rhyne,} \mathrm{MD}^{1,2}$, \\ Maya O'Neil, $\mathrm{PhD}^{3}$, Karli Kondo, $\mathrm{PhD}^{3}$, Rose Relevo, MLIS, $\mathrm{MS}^{3}$, Makalapua Motu'apuaka, $\mathrm{BS}^{3}$, Michele Freeman, $\mathrm{MPH}^{1,3}$, \\ Honora Englander, MD2
}

${ }^{1}$ Department of Medicine, VA Portland Health Care System, Portland, Oregon; ${ }^{2}$ Department of Medicine, Oregon Health and Science University, Portland, Oregon; ${ }^{3}$ Portland VA Evidence-based Synthesis Program, Portland, Oregon; ${ }^{4}$ Consultant in Healthcare Safety and Quality, Baltimore, Maryland.

BACKGROUND: Health systems are faced with a large array of transitional care interventions and patient populations to whom such activities might apply.

PURPOSE: To summarize the health and utilization effects of transitional care interventions, and to identify common themes about intervention types, patient populations, or settings that modify these effects.

DATA SOURCES: PubMed and Cochrane Database of Systematic Reviews (January 1950-May 2014), reference lists, and technical advisors.

STUDY SELECTION: Systematic reviews of transitional care interventions that reported hospital readmission as an outcome.

DATA EXTRACTION: We extracted transitional care procedures, patient populations, settings, readmissions, and health outcomes. We identified commonalities and compiled a narrative synthesis of emerging themes.

DATA SYNTHESIS: Among 10 reviews of mixed patient populations, there was consistent evidence that enhanced discharge planning and hospital-at-home interventions reduced readmissions. Among 7 reviews in specific patient populations, transitional care interventions reduced readmission in patients with congestive heart failure and general medical populations. In general, interventions that reduced readmission addressed multiple aspects of the care transition, extended beyond hospital stay, and had the flexibility to accommodate individual patient needs. There was insufficient evidence on how caregiver involvement, transition to sites other than home, staffing, patient selection practices, or care settings modified intervention effects.

CONCLUSIONS: Successful interventions are comprehensive, extend beyond hospital stay, and have the flexibility to respond to individual patient needs. The strength of evidence should be considered low because of heterogeneity in the interventions studied, patient populations, clinical settings, and implementation strategies. Journal of Hospital Medicine 2016;11:221-230. (c) 2015 Society of Hospital Medicine.
Transitional care has been defined as "a set of actions designed to ensure the coordination and continuity of healthcare as patients transfer between different locations or different levels of care within the same location." ${ }^{1}$ Early studies showed that nurse-led transitional care interventions beginning in the hospital and continuing after discharge had the potential to reduce the rate of hospital readmissions. ${ }^{2,3}$ Since then, the healthcare landscape has been evolving in important ways, with the spread of the electronic medical record, the patient-centered medical home, and an increased push to health systems integration. ${ }^{4-6}$

The potential success or failure of transitional care interventions, which are inherently complex and can

\footnotetext{
*Address for correspondence and reprint requests: Devan Kansagara MD, Portland Veterans Affairs Medical Center, Mailcode: RD71, 3710 SW US Veterans Hospital Rd., Portland, OR 97239; Telephone: 503-2208262; Fax: 503-273-5374; E-mail: kansagar@ohsu.edu

Additional Supporting Information may be found in the online version of this article.

Received: May 26, 2015; Revised: September 18, 2015; Accepted: September 30, 2015

2015 Society of Hospital Medicine DOI 10.1002/jhm.2502

Published online in Wiley Online Library (Wileyonlinelibrary.com).
}

involve multiple components, may depend on the nature of the interventions themselves, the settings in which they were implemented, and/or the populations included. Health systems are faced with a large array of transitional care interventions and patient populations to whom such activities might apply.

The main aim of this article, culled from a larger report commissioned by the Veterans Health Administration $(\mathrm{VHA})^{7}$ was to catalogue which types of transitional care interventions hold promise and which populations have been best studied, to help health systems guide prioritization and adaptation of the most relevant transitional care activities and help focus future research efforts.

\section{METHODS}

We conducted a review of systematic reviews published in English, following Preferred Reporting Items for Systematic Reviews and Meta-analyses reporting guideline for systematic reviews. ${ }^{8}$ A protocol describing the review plan was posted to a public website before the study was initiated. ${ }^{9}$ From an initial review of the literature, we recognized that most systematic reviews typically either examined different transitional 


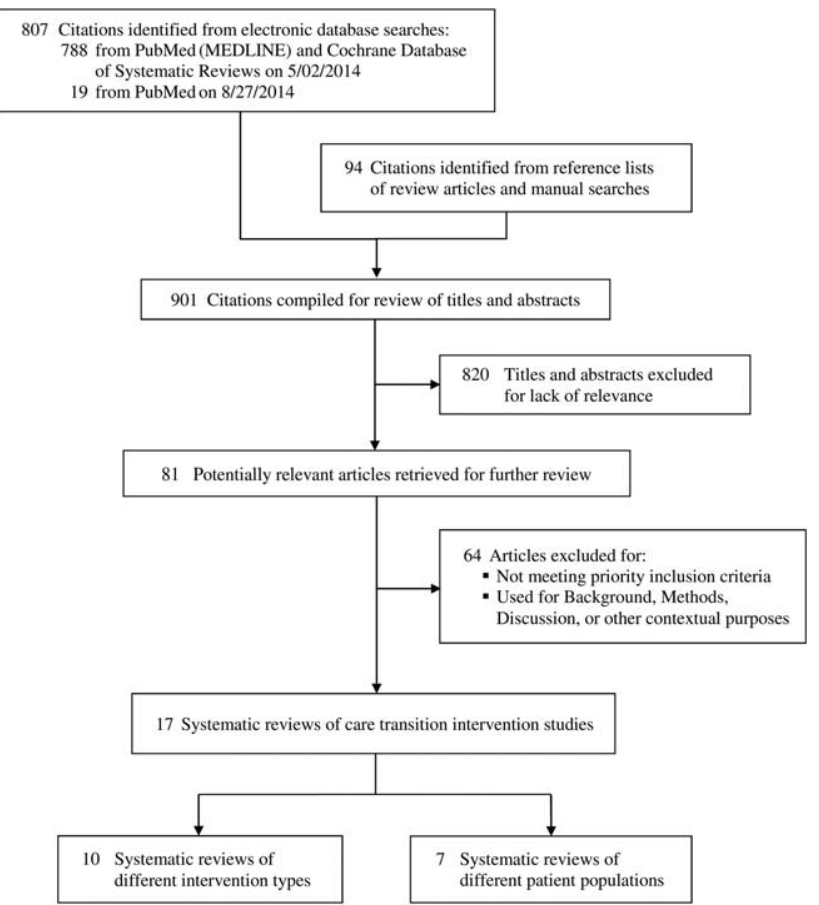

FIG. 1. Literature flow diagram.

care intervention types in a given patient population, or examined a given intervention type in a variety of patient populations. We use the term intervention type to refer to single- or multicomponent interventions that used a similar approach or bundle of care processes (eg, telemonitoring, hospital-at-home), or addressed a similar key process of the care transition (eg, medication reconciliation). Patient populations are defined according to clinical condition (eg, congestive heart failure) or demographic characteristics (eg, geriatric). Given that the review was originally commissioned by the VHA, we excluded pediatric and obstetric patient populations.

We identified categories of patient populations and intervention types with input from a panel of content experts, an initial scan of the literature, and with input from our study team to help guide our literature search (see Supporting Information, Appendix A, in the online version of this article). We searched PubMed and Cochrane databases of systematic reviews from database inception through May 2014.

We selected reviews that reported hospital readmissions as an outcome, regardless of whether it was the primary outcome. However, we summarized other outcomes reported by each review. Within each patient population or intervention type of interest, we first identified reviews that fulfilled key quality criteria: (1) clearly reported their search strategy, (2) reported inclusion and exclusion criteria, and (3) conducted an appraisal of the internal validity of the included trials. ${ }^{10,11}$ If there was more than 1 review within each category fulfilling these criteria, we prioritized the most recent review and those with the broadest scope. We discussed the ultimate choice of review as a group and resolved any disagreements through consensus. One author abstracted prespecified data from each review and a second author checked entries for accuracy (see Supporting Information, Appendix B, in the online version of this article).

We qualitatively synthesized the literature, using the categories of intervention type, patient population, and healthcare setting to organize our synthesis. We further identified common themes that cut across different intervention types and patient populations related to the following characteristics (derived from an existing taxonomy): ${ }^{12}$ transition type (hospital to home, hospital to nursing facility), intervention target (patient, caregiver), key processes (education, personal health record), key personnel involved (nurse, social worker), method of postdischarge follow-up (phone, home visits), and intensity and complexity. We developed brief narrative summaries of findings for each review. These narratives were compiled into a single document and reviewed independently by each of the authors of this report, who then compiled a brief list of key cross-cutting themes in the evidence.

\section{RESULTS}

We reviewed 807 titles and abstracts from the electronic search, and identified an additional 94 from reviewing reference lists and performing manual searches for recently published and unpublished or ongoing studies (Figure 1). Eighty-one systematic reviews met our inclusion criteria and, of these, we selected 17 that were the most recent and broadly scoped: 10 of intervention types (Table 1) and 7 of patient populations (Table 2).

\section{Intervention Types}

Among reviews focused on specific intervention types (Table 1), several show promise in reducing readmissions and/or mortality. ${ }^{13-16}$ There is moderate-strength evidence that structured and individually tailored discharge planning reduces readmissions within 90 days (relative risk [RR]: $0.82,95 \%$ confidence interval [CI]: 0.73 to 0.92$)$ and hospital length of stay $(-0.91$ days, $95 \%$ CI: -1.55 to -0.27$).{ }^{13}$ However, most of the benefit was seen among studies of robust interventions that included a combination of care processes. In 9 of the interventions, a nurse "advocate" helped with discharge planning activities and care coordination. Twelve of the interventions included postdischarge follow-up.

Moderate strength evidence from 61 trials found that hospital-at-home interventions were associated with reductions in 30-day readmissions (RR: 0.75 , 95\% CI: 0.59 to 0.95 ) and mortality (RR: $0.81,95 \%$ CI: 0.69 to 0.95). ${ }^{14}$ Frequently, specific components of the included interventions were not well described, and periods of observation for outcomes were not specified. Interventions were associated with greater patient and caregiver 


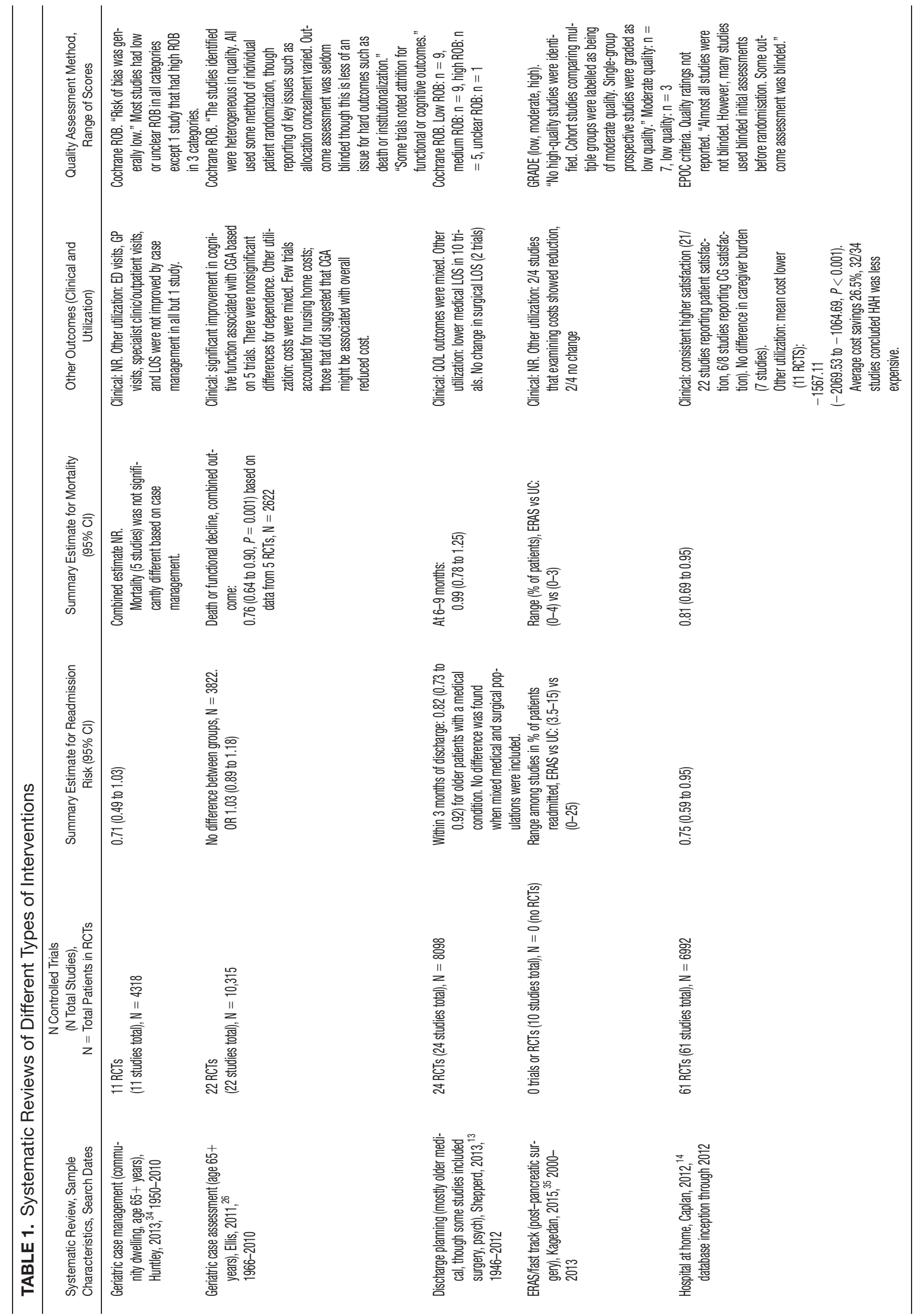




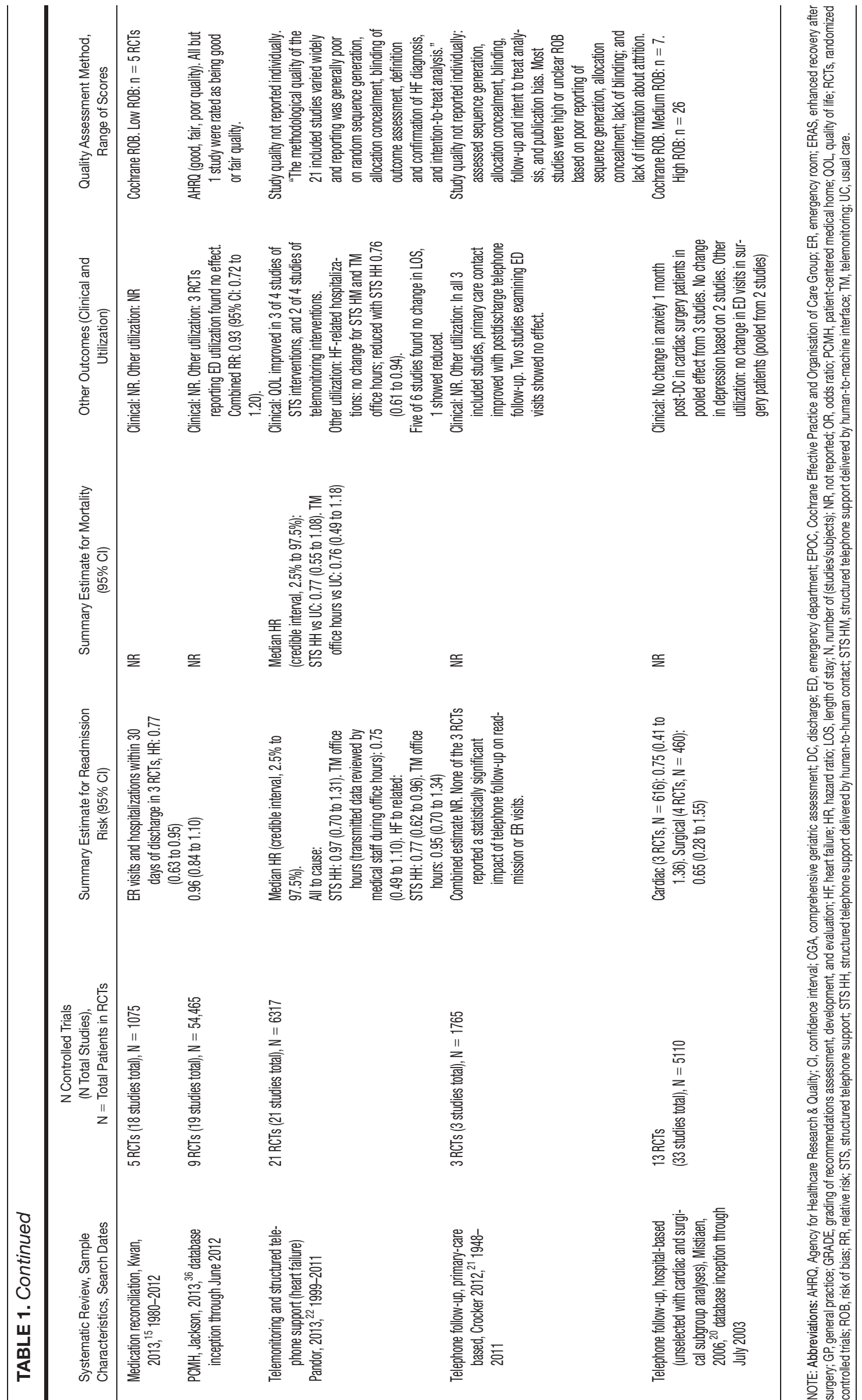




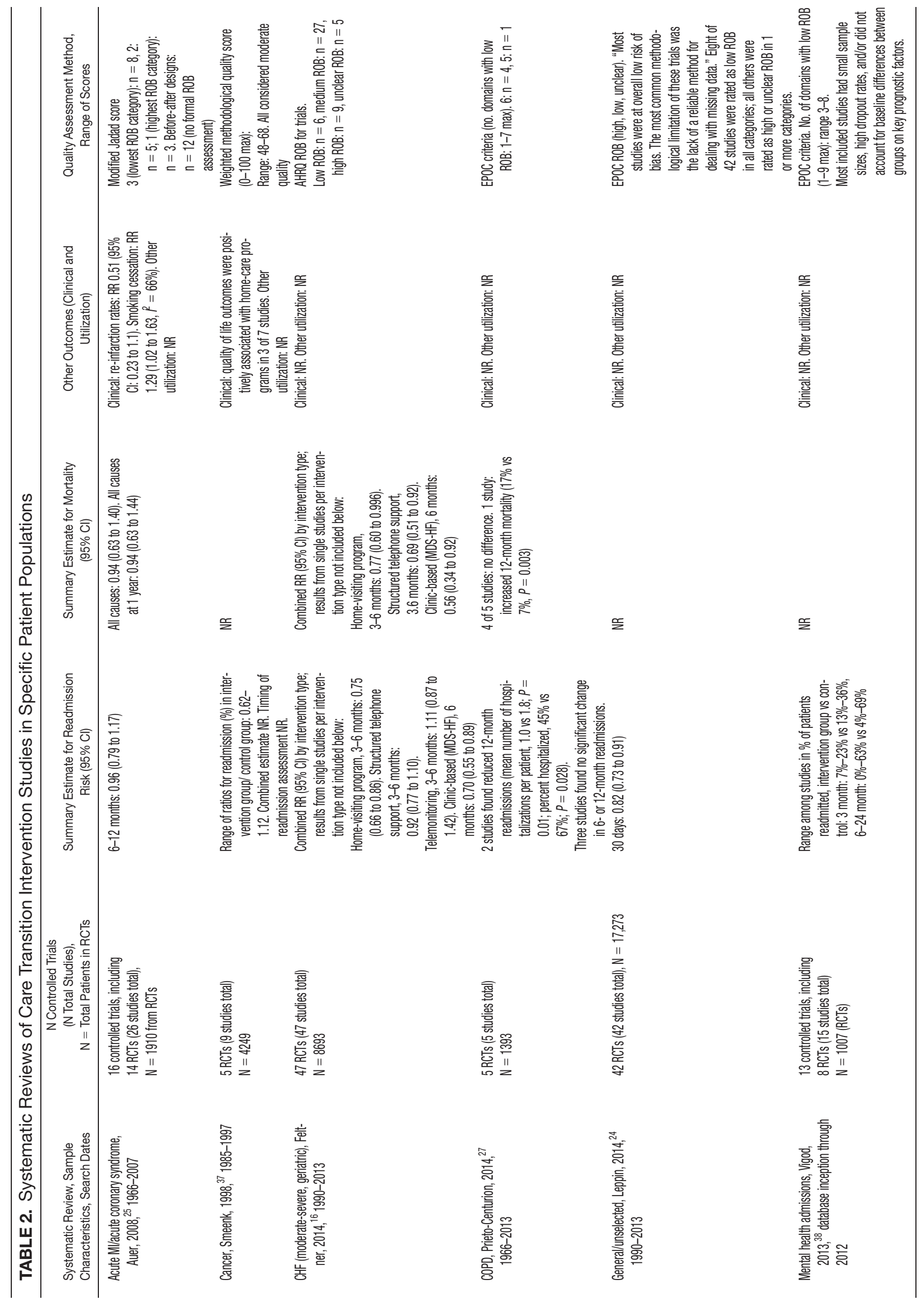


satisfaction in the vast majority of studies reporting such outcomes.

The impact of medication reconciliation interventions on clinically significant adverse drug events was variable. ${ }^{15}$ Readmissions and emergency room visits were reduced (RR: $0.77,95 \%$ CI: 0.0 .63 to 0.95 ) in 3 trials, but this reduction was driven by 1 intervention that included additional care processes such as postdischarge follow-up. ${ }^{17}$ Interventions focused solely on medication reconciliation around the time of discharge were not effective.

One review of patients with stroke or myocardial infarction (MI) described 5 intervention types: hospitalbased discharge preparation, hospital-based patient and family education, community-based patient and family education, community-based models of support interventions, and chronic disease management models of care. ${ }^{18}$ They found moderate-strength evidence that early supported discharge of stroke patients (short hospital stay followed by intensive home care with a multidisciplinary team) shortened length of stay without adversely impacting readmissions or mortality. Specialty care after an MI was associated with reduced mortality, but the strength of evidence was low, being largely based on 1 Veterans Affairs observational study. ${ }^{19}$ There was insufficient evidence examining the other types of interventions in this review.

Two reviews examined the effects of postdischarge follow-up calls in unselected populations. An older Cochrane review from 2006 focused on calls performed by hospital-based personnel. ${ }^{20}$ Though 33 studies including 5110 patients were included in this review, there was inconclusive evidence of the effectiveness of these interventions, largely because of methodological limitations in most included studies. A more recent review similarly concluded there was insufficient evidence of the effects of postdischarge calls on utilization in 3 studies, though they did find that the interventions were associated with higher rates of primary care engagement. ${ }^{21}$

One review focused on postdischarge remote monitoring in patients with congestive heart failure $(\mathrm{CHF})^{22,23}$ via structured telephone support (STS) or telemonitoring. STS interventions typically included periodic scripted telephone calls from nurses to review symptoms, interval physiologic data such as weight, and self-management skills. Telemonitoring focused on remote transfer of physiologic data, with phone contact when abnormal vital signs or weights occurred. STS interventions reduced long-term $(\geq 6$ months), but not short-term (2-3 months) heart failure readmissions, and were associated with reduced long-term mortality. ${ }^{16,23}$ Though 1 review noted a trend toward reduced mortality with telemonitoring interventions, both reviews noted the substantial methodological shortcomings of this literature and the inconsistency of results across studies. There was 
insufficient evidence of the comparative effectiveness between STS and telemonitoring interventions. ${ }^{16}$

One review of CHF patients categorized interventions into 6 types: home-visiting programs, STS, telemonitoring, outpatient clinic-based (including multidisciplinary CHF clinics), primarily educational, and other. ${ }^{16}$ This review found moderate-strength evidence that interventions with multidisciplinary heart failure (HF) clinic visits or home visits reduced both all-cause readmissions and mortality, with number needed to treat below 10 for readmission and 18 to 33 for mortality (for multidisciplinary heart failure clinic and home visiting programs, respectively). STS interventions produced a similar mortality benefit but did not reduce all-cause readmissions.

\section{Healthcare Setting}

We found no evidence directly examining whether intervention effectiveness depends on factors such as the presence of a shared electronic medical record, access to community resources, integration of primary and hospital care, and the presence of a medical home. Moreover, the transitional care literature generally has provided only scant descriptions of the health system context of the interventions.

\section{Patient Population}

The relative importance of careful patient selection, as compared to intervening on an unselected group of patients, is unclear. Many studies in these reviews used inclusion criteria that selected patients who were at high risk for readmission because of older age, significant medical comorbidity, and/or a history of high utilization. However, few reviews explicitly examined variation of intervention effects based on patient criteria.

The characteristics and findings of reviews of specific patient populations are shown in Table 2. One review found studies that did and did not use highrisk patient selection criteria had similar results. ${ }^{15} \mathrm{~A}$ metaregression of trials including general medical or CHF populations did not find significantly different effects between studies without age restrictions and those that included only patients over 65 years of age (interaction $P=0.24$ ). ${ }^{24}$ Similarly, a review of hospital-at-home studies did not find a clear difference in effects among studies in patients younger than 70 years old, between ages 70 and 73 years, and older than 74 years. ${ }^{14}$

Some of the reviews also speculated that focusing on specific groups of patients allowed disease-specific customization of interventions and supported expertise development. For example, 1 review found that interventions in acute MI patients, which focused on effective use of disease-specific medications, were associated with a mortality benefit, though this was largely driven by 1 study. ${ }^{25}$ Another review examining comprehensive geriatric assessment interventions found that gains in the combined outcome of mortality and functional decline were only associated with interventions delivered in a geriatric ward setting. ${ }^{26}$ The authors speculate that the multidisciplinary team of providers developed more expertise and facility with the patient population.

We found insufficient evidence to determine whether transitional care affects specific patient populations differently. Although there were successful interventions in CHF patients and no consistent evidence of benefit in chronic obstructive pulmonary disease (COPD) patients, it is unclear whether these differences were due to the markedly different types of interventions examined or to the choice of population itself. ${ }^{16,27}$ Populations with chronic medical illnesses were well represented in the literature, although there was a dearth of evidence in mental illness or surgical populations.

\section{Cross-cutting Themes}

Across different intervention types, patient populations, and settings, successful interventions tended to be more comprehensive, involve more aspects of the care transition, and include components before and after hospital discharge. Successful interventions also tended to be flexible enough to accommodate individual patient needs. However, the strength of evidence supporting these overarching conclusions should be considered low because these are indirect, post hoc comparisons across literature that includes many different intervention types, studied in varied populations and clinical settings, and implemented in different ways. We found very few comparative effectiveness studies among the included reviews.

As noted above, the effective discharge planning and medication reconciliation interventions were those that included additional personnel and spanned care settings. ${ }^{13,17}$ In contrast, interventions in COPD populations did not consistently reduce readmissions or mortality, but the interventions began after hospital discharge and frequently omitted some care processes such as discharge planning that are often 1 component of successful interventions in other populations. ${ }^{27}$

One review created a "comprehensive support" variable that was based on number of patient interactions, number of personnel involved, number of intervention components, and the ability of the intervention to address self-management needs. ${ }^{24} \mathrm{~A}$ metaregression including 42 trials, the vast majority of which included general medical patients or patients with $\mathrm{CHF}$ and were considered to be methodologically sound, found interventions were overall associated with reductions in readmissions (pooled RR: 0.82, 95\% CI: 0.73 to 0.91 ), and interventions with the most comprehensive support accounted for most of the benefit ( $R R$ readmission in the 7 studies with highest comprehensive support scores compared to 15 studies with the lowest scores: $0.63,95 \%$ CI: 0.43 to 0.91$).^{24}$ 
In a review of 47 trials in CHF patients, the key processes of care that seemed to be associated with reduced readmissions included: self-management education delivered in person, early postdischarge contact, a point of postdischarge contact, and the ability to individually tailor the intervention. ${ }^{16}$

It is unclear whether home visits are a necessary component of transitional care interventions. A metaanalysis of trials including general medicine or CHF patients did not find that the setting of care delivery influenced outcomes; however, all but 1 of the most comprehensive interventions included home visits in their model. ${ }^{24} \mathrm{~A}$ review of CHF populations found interventions with multidisciplinary HF clinic visits or home visits reduced all-cause readmissions and mortality, but found insufficient evidence directly comparing interventions with and without home visits. ${ }^{16}$

We found little evidence examining the impact of different transition types (most studies focused on hospital-to-home transitions), intervention targets (most studies focused on patients rather than caregivers), or key personnel involved.

\section{DISCUSSION}

We examined 17 systematic reviews across different patient populations representing a variety of intervention types to provide a broad overview of the care transitions literature. Variations in population studied, intervention definition, personnel, outcome definition, and setting make it difficult to identify strong evidence in support of a specific intervention type that should be broadly implemented. There were, however, some common themes that emerged across the literature suggesting that successful interventions addressed more aspects of the care transition, included the means to assess and respond to individual peridischarge needs, and included components that spanned care settings. In practical terms, the actualization of these themes has been accomplished in many interventions with the addition of transitional care personnel such as nurses and/or pharmacists. Additionally, interventions have often been tailored to the needs of individual patients with the use of needs assessment and patient-centered personalized health records. ${ }^{1}$

Because there are many potential steps in the care transition, focusing on only 1 of these steps, such as medication reconciliation, is unlikely to have significant benefit on risk of readmission. ${ }^{15}$ The pathways to readmission vary, as suggested both by the inability to accurately anticipate which patients will be readmitted, ${ }^{28}$ and by case review studies characterizing underlying factors contributing to preventable readmissions. ${ }^{29}$

The problems with recommending that a specific intervention be broadly implemented include both the lack of evidence supporting such a recommendation and the likelihood that the transitional care gaps are not the same in all settings, or for all populations of patients treated. As health systems rapidly evolve, it may be useful for them to inventory strengths and weaknesses of their current approach to transitional care both to identify critical care gaps and to avoid investment in resource-intensive transitional care interventions that may be redundant with existing activities.

Indeed, transitional care gaps may have changed over the last decade. Two large reviews showed that more recently published studies were less likely to have found an improvement in outcomes. ${ }^{14,24}$ In the years since some of the most successful and widely cited transitional care interventions were developed and evaluated, many health systems have undertaken major transformations, including the adoption of the patient-centered medical home model and integration of electronic health records, which may implicitly address some earlier gaps. For instance, foundational qualitative work for the Care Transitions Measure identified discontinuities in information transfer as 1 of 4 major transitional care barriers identified by patients, and the personal health record was created, in part, to address this gap. ${ }^{30} \mathrm{~A}$ shared electronic health record across healthcare settings has the potential to mitigate some of these concerns.

In general, there is an overarching need for better evidence to guide selection and implementation of complex, multicomponent transitional care interventions in different settings. There remain a number of gaps regarding the operationalization of interventions. For instance, the optimal choice of personnel, the comparative effects of home visits and other forms of postdischarge follow-up, and the best approach to patient selection (whether through use of a formal readmission risk assessment model or a focus on populations with high-risk comorbidities) are unknown.

One of the major weaknesses of the transitional care literature is the marked variation in intervention definitions, timing of outcome follow-up, and descriptions of interventions and usual care. Use of taxonomies to guide study design and description may help standardize reporting.

Most of the care transitions literature has been hospital-focused, and the interventions often extend hospital services beyond hospitalization. Given the growth of medical homes, it will be important to examine the effectiveness of outpatient-based care transitions models that "reach-in" to the hospital. Studies comparing approaches such as home-visit and telephone-based interventions, different risk-prioritization schemes, and the use of different types of personnel are also needed.

There is very little literature examining transitional care interventions in patients with mental health conditions or undergoing surgery. A recent report for the Veterans Health Administration found that $24 \%$ of patients with chronic mental health conditions are readmitted within 30 days of discharge. ${ }^{31}$ About 1 in 7 Medicare patients admitted to a surgical service is readmitted within 30 days. $^{32}$ The transitional care 
needs of these populations may differ substantially from medical populations and warrant further study.

Our review has a number of important limitations. Our overview of the literature was necessarily broad rather than in-depth. There are many nuances in the results, internal validity, and generalizability of studies that are not represented in our overview. It was difficult to use established criteria to formally rate the strength of evidence for each of our conclusions, but we indicated strength of evidence ratings when reported in reviews. As we note in the results, our assessment of cross-cutting themes is based largely on low-strength evidence, given the indirect comparisons and the many factors that varied among the included studies. Our inclusion criteria specified readmissions as an outcome, but there are care transitions that focus exclusively on other outcomes, such as smoking cessation interventions around the time of discharge. ${ }^{33}$ Furthermore, there are many outpatient-based interventions designed to affect emergency room and hospital utilization that are not captured in our review, but may nevertheless be important to understanding the role of care coordination in the context of the medical home. We did not systematically update the included reviews' searches, and there may be more recent studies not represented here, though we are not aware of newer studies that would substantively change our summary of findings.

\section{CONCLUSIONS}

The literature includes many different types of interventions, studied in varied populations and clinical settings, and implemented in different ways. Furthermore, there are very little comparative effectiveness data. It is therefore difficult to conclusively identify specific intervention components and characteristics that are necessary for successful care transitions. Effective interventions are generally more comprehensive, address more aspects of the care transition, extend beyond the hospital stay, and have the flexibility to respond to individual patient needs. Transitional care interventions have not been well studied in integrated health system settings, or in mental health and surgical populations.

Disclosures: The views expressed in this article are those of the authors and do not necessarily represent the views of the US Department of Veterans Affairs or the US government.

The research reported here was supported by the Department of Veterans Affairs, Veterans Health Administration (VHA) Project ESP 05-225, VA\#01-0206. Dr. Jencks' work on this project was supported in part by a grant from the Quality Enhancement Research Initiative (05-225), Department of Veterans Affairs. Dr. Jencks has reported prior consulting work with the following entities: Inovalon, Care Centrix, Affymax, Curaspan, Reinforced Care, Health Services Advisory Group, Delmarva Foundation, Connecticut Peer Review Organization, Maryland Health Services Cost Review Commission, Institute for Healthcare Improvement, American Association for Respiratory Care, Monaghan Medical, Iowa Society for Respiratory Care.

\section{References}

1. Coleman EA. Falling through the cracks: challenges and opportunities for improving transitional care for persons with continuous complex care needs. J Am Geriatr Soc. 2003;51(4):549-555.
2. Coleman EA, Parry C, Chalmers S, Min S-J. The care transitions intervention: results of a randomized controlled trial. Arch Intern Med. 2006;166(17):1822-1828.

3. Naylor MD, Brooten D, Campbell R, et al. Comprehensive discharge planning and home follow-up of hospitalized elders: a randomized clinical trial. JAMA. 1999;281(7):613-620.

4. Hsiao CJ, Hing E. Use and characteristics of electronic health record systems among office-based physician practices: United States, 20012013. NCHS Data Brief. 2014(143):1-8.

5. Robeznieks A. Reform Update: Medical-home adoption growing; evidence of effectiveness still elusive. Modern Healthcare website. Available at: http://www.modernhealthcare.com/article/20140818/NEWS/ 308189963. Published August 18, 2014. Accessed April 14, 2015.

6. Enthoven AC. Integrated delivery systems: the cure for fragmentation. Am J Manag Care. 2009;15(10 suppl):S284-S290.

7. Kansagara D, Chiovaro JC, Kagen D, et al. Transitions of care from hospital to home: a summary of systematic evidence reviews and recommendations for transitional care in the Veterans Health Administration. VA-ESP Project \#05-225. Available at: http://www.ncbi.nlm. nih.gov/pubmedhealth/PMH0078978. Accessed August 1, 2015.

8. Moher D, Liberati A, Tetzlaff J, Altman DG, The PRISMA Group (2009). Preferred reporting items for systematic reviews and metaanalyses: the PRISMA statement. PLoS Med. 2009;6(7):e1000097.

9. Health Services Research \& Development. ESP reports in progress. Available at: http://www.hsrd.research.va.gov/publications/esp/in_ progress.cfm. Accessed April 14, 2015.

10. Shea BJ, Grimshaw JM, Wells GA, et al. Development of AMSTAR: a measurement tool to assess the methodological quality of systematic reviews. BMC Med Res Methodol. 2007;7(1):10.

11. Whitlock EP, Lin JS, Chou R, Shekelle P, Robinson KA. Using existing systematic reviews in complex systematic reviews. Ann Intern Med. 2008;148(10):776-782.

12. Olson DM, Prvu Bettger J, Alexander KP, et al. Transition of care for acute stroke and myocardial infarction patients from hospitalization to rehabilitation, recovery, and secondary prevention. Evidence Reports/Technology Assessments, No. 202. Report No.: 11(12)-E011. Rockville, MD: Agency for Healthcare Research and Quality; 2011. Available at: http://www.ncbi.nlm.nih.gov/books/NBK82455. Accessed August 1, 2015.

13. Shepperd S, Lannin NA, Clemson LM, McCluskey A, Cameron ID, Barras SL. Discharge planning from hospital to home. Cochrane Database Syst Rev. 2013;1:CD000313.

14. Caplan GA, Sulaiman NS, Mangin DA, Aimonino Ricauda N, Wilson $\mathrm{AD}$, Barclay L. A meta-analysis of "hospital in the home". Med J Aust. 2012;197(9):512-519.

15. Kwan JL, Lo L, Sampson M, Shojania KG. Medication reconciliation during transitions of care as a patient safety strategy: a systematic review. Ann Intern Med. 2013;158(5 pt 2):397-403.

16. Feltner C, Jones CD, Cene CW, et al. Transitional care interventions to prevent readmissions for persons with heart failure: a systematic review and meta-analysis. Ann Intern Med. 2014;160(11):774-784.

17. Jack BW, Chetty VK, Anthony D, et al. A reengineered hospital discharge program to decrease rehospitalization: a randomized trial. Ann Intern Med. 2009;150(3):178-187.

18. Prvu Bettger J, Alexander KP, Dolor RJ, et al. Transitional care after hospitalization for acute stroke or myocardial infarction: a systematic review. Ann Intern Med. 2012;157(6):407-416.

19. Ho PM, Luther SA, Masoudi FA, et al. Inpatient and follow-up cardiology care and mortality for acute coronary syndrome patients in the Veterans Health Administration. Am Heart J. 2007;154(3):489-494.

20. Mistiaen P, Poot E. Telephone follow-up, initiated by a hospital-based health professional, for postdischarge problems in patients discharged from hospital to home. Cochrane Database Syst Rev. 2006(4):CD004510.

21. Crocker JB, Crocker JT, Greenwald JL. Telephone follow-up as a primary care intervention for postdischarge outcomes improvement: a systematic review. Am J Med. 2012;125(9):915-921.

22. Pandor A, Gomersall T, Stevens JW, et al. Remote monitoring after recent hospital discharge in patients with heart failure: a systematic review and network meta-analysis. Heart. 2013;99(23):1717-1726.

23. Pandor A, Thokala P, Gomersall T, et al. Home telemonitoring or structured telephone support programmes after recent discharge in patients with heart failure: systematic review and economic evaluation. Health Technol Assess. 2013;17(32):1-207, v-vi.

24. Leppin AL, Gionfriddo MR, Kessler M, et al. Preventing 30-day hospital readmissions: a systematic review and meta-analysis of randomized trials. JAMA Intern Med. 2014;174(7):1095-1107.

25. Auer R, Gaume J, Rodondi N, Cornuz J, Ghali WA. Efficacy of inhospital multidimensional interventions of secondary prevention after acute coronary syndrome: a systematic review and meta-analysis. Circulation. 2008;117(24):3109-3117.

26. Ellis G, Whitehead MA, Robinson D, O'Neill D, Langhorne P. Comprehensive geriatric assessment for older adults admitted to hospital: meta-analysis of randomised controlled trials. BMJ. 2011;343:d6553.

27. Prieto-Centurion V, Markos MA, et al. Interventions to reduce rehospitalizations after chronic obstructive pulmonary disease exacerbations. A systematic review. Ann Am Thorac Soc. 2014;11(3):417-424. 
28. Kansagara D, Englander H, Salanitro A, et al. Risk prediction models for hospital readmission: a systematic review. JAMA. 2011;306(15): 1688-1698.

29. Oddone EZ, Weinberger M, Horner M, et al. Classifying general medicine readmissions. J Gen Intern Med. 1996;11(10):597-607.

30. Coleman EA, Mahoney E, Parry C. Assessing the quality of preparation for posthospital care from the patient's perspective: the care transitions measure. Med Care. 2005;43(3):246-255.

31. Carey K, Stefos T. An Investigation Into the Cost of VA Hospital Readmissions. Washington DC: US Department of Veterans Affairs, Office of Quality, Safety and Value; 2014.

32. Tsai TC, Joynt KE, Orav EJ, Gawande AA, Jha AK. Variation in surgical-readmission rates and quality of hospital care. N Engl J Med. 2013;369(12):1134-1142.

33. Rigotti NA, Regan S, Levy DE, et al. Sustained care intervention and postdischarge smoking cessation among hospitalized adults: a randomized clinical trial. JAMA. 2014;312(7):719-728.
34. Huntley AL, Thomas R, Mann M, et al. Is case management effective in reducing the risk of unplanned hospital admissions for older people? A systematic review and meta-analysis. Fam Pract. 2013;30(3): 266-275.

35. Kagedan DJ, Ahmed M, Devitt KS, Wei AC. Enhanced recovery after pancreatic surgery: a systematic review of the evidence. HPB (Oxford). 2015;17(1):11-16.

36. Jackson GL, Powers BJ, Chatterjee R, et al. Improving patient care. The patient centered medical home. A systematic review. Ann Intern Med. 2013;158(3):169-178.

37. Smeenk FW, van Haastregt JC, de Witte LP, Crebolder HF. Effectiveness of home care programmes for patients with incurable cancer on their quality of life and time spent in hospital: systematic review. BMJ. 1998;316(7149):1939-1944.

38. Vigod SN, Kurdyak PA, Dennis CL, et al. Transitional interventions to reduce early psychiatric readmissions in adults: systematic review. Br J Psychiatry. 2013;202(3):187-194. 